\title{
Neurophysiology in urogynaecology
}

\author{
Peter L. Dwyer
}

Published online: 7 October 2011

(C) The International Urogynecological Association 2011

In this issue of the International Urogynecology Journal, there is a focus on neurophysiology in urogynaecology. Neurophysiological studies have provided considerable advances in our understanding of the pathophysiology of pelvic floor disorders including urinary incontinence and pelvic organ prolapse. These studies have shown that there is a neurogenic basis for stress incontinence [1] and uterovaginal prolapse [2] caused by vaginal delivery as well as other associated factors of weakness of the pelvic floor muscles and connective tissue. A study by Kenton et al. [3] again confirms the value of neurological investigations in the study of pelvic floor disorders. Using concentric needle electromyography, they showed that women with stress incontinence had poorer urethral innervation than continent controls. They also found that there were significant neuropathic changes with ageing which is consistent with lower urethral pressures and decreasing numbers and diameter of striated muscles fibres of the urethral sphincter and pubococcygeus muscle with increasing age.

Although neurophysiological investigations have contributed to our understanding of causation of urinary incontinence and prolapse, they have not to date been shown to have a significant role in the routine investigation and management of female urinary incontinence or uterovaginal prolapse. With this in mind, we have asked David Vodušek to provide an overview of the role of neurophysiological investigations in urogynaecology today. In his review [4], he discusses what neurophysiological tests tell

\section{P. L. Dwyer $(\bowtie)$}

Department of Urogynecology, Mercy Hospital for Women,

University of Melbourne,

Melbourne, VIC, Australia

e-mail: pdwyer@connexus.net.au us about nerves and muscles and what neurological investigations we should perform and when.

Chronic pelvic pain in women is a common and difficult problem affecting one in five women, with most not having a clearly defined cause [5]. Many women date their pain back to gynaecological surgery, so not surprisingly, this is also an area of high medical litigation and anxiety to pelvic surgeons. The study of Possover et al. [6] in this issue reports on 95 consecutive women with pelvic pain and/or bladder or bowel dysfunction following pelvic prolapse surgery. These women underwent laparoscopic exploration and neurolysis for what the authors considered to be iatrogenic pelvic nerve injury. The authors' conclusions are that prolapse surgery is a common cause of nerve injury and pelvic pain, which is frequently underdiagnosed but can be successfully treated surgically.

Many women with chronic pelvic pain often present having already been told that their pain is caused by surgical nerve injury or entrapment. This explanation is very attractive to women with chronic pain as it gives them a clear understandable cause for their pain and hope that it can be successfully treated by surgical exploration; but how true is this? The causes, diagnosis and management of pudendal neuralgia have recently been reviewed by Stav et al. [7] including the role of neurophysiological studies and nerve decompression surgery. Neurophysiological tests were considered to have a low diagnostic value in the diagnosis of neuropathic pain and to be complementary investigations, which is in line with what Vodušek explains about the limitations of these tests [4]. Pudendal neuralgia was considered a clinical syndrome rather than a specific diagnosis, and "there is no evidence to support equating the presence of this syndrome with a diagnosis of pudendal nerve entrapment, although that may be one etiological condition". Such caution is probably appropriate also when 
ascribing persistent postoperative pain in other urogynaecological conditions to nerve involvement.

Surgical nerve decompression of the pudendal nerve can be performed via a transperineal, transgluteal, and transvaginal approach. The results of relieving pain seem to be similar between the different approaches and range from $50 \%$ to $86 \%$ [7]. Stav et al. [7] concluded that as no randomized controlled studies were available and with the significant placebo effect in pain syndromes, it was difficult to rate the effectiveness of any of these surgical decompression techniques.

Pain after prolapse surgery does occur. Gluteal pain was described by Cruikshank [8] after the sacrospinous fixation in $15 \%$ of their patients, and he considered this pain was caused by dissection into the perirectal space. Sze and Karram [9] thought that this postoperative pain was due to injury to nerves in the coccygeal sacrospinous ligament complex. Maher et al. [10] found that gluteal pain of a similar nature occurred in $19 \%$ of cases following iliococcygeal fixation compared to $14 \%$ in the sacrospinous group. In this series, delayed absorbable sutures were used, and the pain resolved spontaneously in all cases by 3 months but was still a considerable cause of concern and dissatisfaction to patients. No patients who had a transvaginal extraperitoneal uterosacral suspension for posthysterectomy prolapse had similar gluteal pain or sciatica after surgery [11]. We believe there are other possible causes of postoperative buttock pain apart from nerve injury or entrapment such as muscular injury secondary to suture fixation and ischaemia.

In some women with chronic pelvic pain following surgery, there is a history of pelvic pain with previous surgery such as laparoscopies performed for endometriosis or pelvic pain. Beware the patient who presents with excessive symptoms of dragging pelvic pain or urinary frequency and urgency which are out of proportion to the degree of prolapse. In my experience, these patients are at high risk of persistent postoperative pelvic pain, which will then be attributed to your repair. If the pain and irritable bladder symptoms are related to the prolapse, a vaginal pessary will improve these symptoms, but when related to other causes, the pessary causes discomfort, and removal is soon requested.
The aetiology of postoperative pelvic pain, the value of neurophysiological testing, and the place of surgery need further clarification. Women with chronic pain require careful assessment and conservative treatment, ideally where possible in a multidisciplinary clinic. Plato's words of 2 millennia ago chastise us still: "The greatest mistake in the treatment of diseases is that there are physicians for the body and physicians for the soul, although the two cannot be separated."

\section{References}

1. Snooks SJ, Setchell M, Swash M, Henry MM (1984) Injury to innervation of pelvic floor sphincter musculature in childbirth. Lancet 2(8402):546-550

2. Smith AR, Hosker GL, Warrell DW (1989) The role of partial denervation of the pelvic floor in the aetiology of genitourinary prolapsed and stress incontinence of urine. A neurophysiological study. Br J Obstet Gynecol 96:24-28

3. Kenton K, Mueller E, Brubaker L (2011) Continent women have better urethral neuromuscular function than those with stress incontinence. Int Urogynecol J. doi 10.1007/s00192-011-1447-7

4. Vodušek DB (2011) The role of clinical neurophysiology in urogynecology. Int Urogynecol J. doi 10.1007/s00192-011-1485-1

5. Zondervan KT, Yudkin PL, Vessey MP, Jenkinson CP, Dawes MG, Barlow DH, Kennedy SH (2001) Chronic pelvic pain in the community-symptoms, investigations, and diagnoses. Am J Obstet Gynecol 184:1149-1155

6. Possover M, Lemos N (2011) Risks, symptoms, and management of pelvic nerve damage secondary to surgery for pelvic organ prolapse: a report of 95 cases. Int Urogynecol J. doi 10.1007/ s00192-011-1539-4

7. Stav K, Dwyer PL, Roberts L (2009) Pudendal neuralgia-fact or fiction? Obstet Gynecol Surv 64:190-199

8. Cruikshank SH (1987) Preventing posthysterectomy vaginal vault prolapse and enterocele during vaginal hysterectomy. Am J Obstet Gynecol 156:1433-1440

9. Sze HM, Karram MM (1997) Transvaginal repair of vault prolapse. Obstet Gynecol 89:466-475

10. Maher C, Murray C, Carey M, Dwyer PL, Agoni AM (2001) Iliococcygeus or sacrospinous fixation for vaginal vault prolapse. Obstet Gynecol 98:40-44

11. Dwyer PL, Fatton B (2008) Bilateral extraperitoneal uterosacral suspension: a new approach to correct post-hysterectomy vaginal vault prolapse. Int Urogynecol J Pelvic Floor Dysfunct 19:283-292 